\title{
5 \\ Auswirkungen von Digitalisierung und KI auf die wissenschaftliche Arbeit
}

\author{
Verena Nitsch und Peter Buxmann
}

Seit Jahrzehnten untersucht die arbeitswissenschaftliche Forschung die Auswirkungen des Digitalisierungstrends auf Beschäftigte und Arbeitsprozesse (siehe auch Mütze-Niewöhner und Nitsch 2020). Empirische Untersuchungen existieren bereits zu zahlreichen Funktionsbereichen (z. B. Produktion und Service) und Berufsgruppen (z. B. Elektro- und Metallberufe). Bislang liegen jedoch kaum empirische Befunde zu den Effekten der Digitalisierung auf die Arbeit von Forschenden vor. Entsprechend schwierig gestaltet sich die Aufgabe, mögliche gegenwärtige und zukünftige Auswirkungen des Digitalisierungstrends und dem Einsatz von KI auf die wissenschaftliche Arbeit und damit Beschäftigte abzuschätzen. Jedoch existieren zahlreiche Befunde zur thematisch verwandten Wissensarbeit. Es lässt sich daher davon ausgehen, dass einige dieser Befunde auf die wissenschaftliche Arbeit übertragbar sind.

Das folgende Kapitel widmet sich daher zunächst der Charakterisierung von wissenschaftlicher Arbeit als spezielle Form der Wissensarbeit. Vor diesem Hintergrund wird anschließend die Evidenzlage zu Auswirkungen der Digitalisierung auf Wissensarbeitende skizziert und eine Abschätzung der Übertragbarkeit der Befunde auf den wissenschaftlichen Beruf vorgenommen. Es wird ferner postuliert, dass der

Dieses Kapitel entstand durch intensive Diskussion und Zusammenarbeit mit der interdisziplinären Projektgruppe „Digitale Arbeitswelten“, der Carl Friedrich Gethmann, Peter Buxmann, Julia Distelrath, Bernhard G. Humm, Stephan Lingner, Verena Nitsch, Jan C. Schmidt und Indra Spiecker genannt Döhmann angehörten.

\footnotetext{
V. Nitsch $(\bowtie)$

Institut für Arbeitswissenschaft, RWTH Aachen, Aachen, Deutschland

E-Mail: v.nitsch@iaw.rwth-aachen.de

P. Buxmann

Fachgebiet Wirtschaftsinformatik, Technische Universität Darmstadt, Darmstadt, Deutschland E-Mail: buxmann@is.tu-darmstadt.de

C Der/die Autor(en) 2022

C. F. Gethmann et al., Künstliche Intelligenz in der Forschung, Ethics of Science and Technology Assessment 48, https://doi.org/10.1007/978-3-662-63449-3_5
} 
Einsatz von KI-Technologien in erster Linie eine zunehmende Automatisierung von informatorischen/kognitiven Routinetätigkeiten in der wissenschaftlichen Arbeit bewirken wird. Dementsprechend werden einige Effekte der Automatisierung solcher Tätigkeiten vorgestellt und mögliche Auswirkungen auf den Arbeitsmarkt und Arbeitstätigkeiten diskutiert. Um ein genaueres Bild zu Digitalisierungseffekten, dem Einsatz von KI-Methoden und der Einstellung von wissenschaftlich Beschäftigten zu KI-Methoden zeichnen zu können, wurde eine Befragung durchgeführt, deren wesentliche Ergebnisse in diesem Kapitel vorgestellt werden. Im weiteren Verlauf des Kapitels werden die Auswirkungen von KI aus einer ökonomischen Perspektive beleuchtet. Das Kapitel schließt ab mit einem kurzen Resümee und einigen Empfehlungen für die Gestaltung von wissenschaftlicher Arbeit im Zeitalter der Digitalisierung und KI.

\subsection{Wissenschaftliche Arbeit als Teilbereich der Wissensarbeit}

Um die Auswirkungen der Digitalisierung und des Einsatzes von KI-Methoden auf die wissenschaftliche Arbeit abschätzen zu können, bedarf es zunächst eines grundlegenden Verständnisses dieser Arbeit. Obgleich in der Fachdisziplin der Arbeitswissenschaft und verwandten Fachbereichen wie der Arbeits- und Organisationspsychologie sowie der Arbeitssoziologie bislang wenig Literatur zur Charakterisierung der wissenschaftlichen Arbeit existiert, beschäftigt sich eine Vielzahl von Expertinnen und Experten bereits seit Jahrzehnten mit der näheren Beschreibung und Erforschung mit der verwandten Thematik der Wissensarbeit.

Mit der seit Mitte des 20. Jahrhunderts beobachteten gesellschaftlichen Entwicklung von einer Industrie- zu einer Wissensgesellschaft und der damit verbundenen verstärkten Ausrichtung auf professionalisierte, technisierte und dienstleistungsorientierte Tätigkeiten, gewann die Wissensarbeit zunehmend an Bedeutung und Beachtung (Nerdinger et al. 2014). Wissensarbeit ist heutzutage in vielen Berufen zu finden, z. B. bei Journalisten, Medizinern, Informatikern und Beratungsdienstleistenden.

Die Verwendung des Begriffs der Wissensarbeit in der deutschen Fachliteratur lässt sich auf die Übersetzung des englischen Begriffs ,knowledge work“ zurückführen, wo er jedoch zumeist breiter ausgelegt wird. Drucker (1991) prägte maßgeblich den Begriff des ,knowledge workers“ und beschrieb diesen als ,an employee whose major contribution depends on his employing his knowledge rather than his muscle power and coordination, frequently contrasted with production workers who employ muscle power and coordination to operate machines" (Drucker 1991, S. 564). Weiterhin definierte Drucker „knowledge workers“ als hoch qualifizierte Fachkräfte, die durch formale Ausbildung erworbenes theoretisches und analytisches Wissen im Beruf anwenden, um neue Produkte oder Dienstleistungen zu entwickeln. Drucker war ferner der Auffassung, dass kontinuierliches Lernen und ständige Innovationsbereitschaft wichtige Voraussetzungen für eine erfolgreiche Wissensarbeit seien. 
Eine häufig zitierte Definition der Wissensarbeit aus dem deutschsprachigen Raum stammt hingegen von Willke (1998). Willke unterscheidet zwischen wissensbasierter Arbeit, die auf spezialisierter Expertise von Personen gründet und in den meisten Formen der Facharbeit vorliegt, und Wissensarbeit, die Tätigkeiten wie Kommunikation, Transaktionen und Interaktionen umfasst und erfordert, dass relevantes Wissen „(1) kontinuierlich revidiert, (2) permanent als verbesserungsfähig angesehen, (3) prinzipiell nicht als Wahrheit sondern als Ressource betrachtet wird und (4) untrennbar mit Nichtwissen gekoppelt ist, sodass mit Wissensarbeit spezifische Risiken verbunden sind.“ (Willke 1998, S. 161).

In Anlehnung an die Definitionen von Drucker und Willke charakterisieren May et al. (2002) die Wissensarbeit etwas näher. Sie spezifizieren unter anderem, dass die Aufgaben- und Problemstellungen bei der Wissensarbeit meist komplex und problemhaltig sind. Für die Problemlösung müssen oftmals neue Vorgehensweisen unter Einbezug aktuellen theoretischen, aber auch kontextuellen Wissens erarbeitet werden, was Kreativität erfordern kann. Letzteres wird gelegentlich auch als Innovationsarbeit bezeichnet. So beschreibt z. B. Hacker (2018) Innovationsarbeit als „Suchen von Lösungen und Wegen zur Lösung bei gut sowie schlecht definierten Problemen“ (Hacker 2018, S. 108).

Während die hier genannten und weitere Definitionen zur Wissensarbeit sich in einigen Aspekten unterscheiden, sind viele sich darin einig, dass die Wissensarbeit mehr umfasst als die Verarbeitung von Informationen und Anwendung von Wissen. Vielmehr ist sie spezifisch gekennzeichnet durch eine hohe Selbstständigkeit und eine gewisse (ergebnis- und prozessbezogene) Unsicherheit; sie ist komplex, kommunikationsintensiv und umfasst routinierte ebenso wie kreative Tätigkeiten, wodurch sich die Wissensarbeit von der klassischen Facharbeit unterscheidet. Die bei anderen Arbeitsformen üblicherweise eingesetzten innerbetrieblichen Kontroll- und Steuerungsformen finden somit bei der Wissensarbeit keine Anwendung (Wilkesmann 2005).

Im Sinne der Definition von Drucker (1991) sind Forschende als hoch qualifizierte Fachkräfte zu bezeichnen, die ihr Wissen anwenden, um neues Wissen oder wissenschaftliche Methoden zu entwickeln. Die Gruppe der Forschenden wäre dementsprechend der Kategorie der Wissensarbeitenden zuzuordnen. Es ist davon auszugehen, dass sich wissenschaftliche Arbeit im Bereich Forschung und Entwicklung durch ein besonders hohes Maß an Wissensarbeit (inkl. Innovationsarbeit) auszeichnet und damit ebenso von hoher Selbstständigkeit, (Prozess-)Unsicherheit und Kommunikationsintensität geprägt ist.

\subsection{Auswirkungen der Digitalisierung auf die Arbeitsbedingungen von wissenschaftlich Arbeitenden}

Die Digitalisierung zeigt in vielen Arbeitsbereichen sowohl positive als auch negative Auswirkungen auf Arbeitsbedingungen und die dadurch beeinflusste Gesundheit und Arbeitszufriedenheit von Beschäftigten. Es ist zu vermuten, dass Digitalisierungsmaßnahmen bei wissenschaftlich Arbeitenden ähnliche Effekte zeigen wie sie bei anderen wissensintensiven Berufsgruppen bereits ermittelt wurden. 
Auf die physische und psychische Gesundheit von Beschäftigten wirkt sich der Digitalisierungstrend insgesamt eher nachteilig aus. Ein Teil der häufig festgestellten negativen gesundheitlichen Auswirkungen von Wissensarbeit lässt sich auf den damit verbundenen hohen Anteil von Büroarbeit an Computerarbeitsplätzen zurückführen. So zeigen arbeitswissenschaftliche Untersuchungen zu Bildschirmarbeit bereits ab einer Arbeitszeit von 2 Stunden diverse Beeinträchtigungen der Augen, darunter Sehschärfeminderungen, Farbsinnstörungen sowie Akkomodations- und Adaptionsstörungen (Köchling 1985). Eine Umfrage von über 7000 Beschäftigten zeigte weiterhin stark verbreitete körperliche Fehlbeanspruchungen und eine deutliche Fehlbeanspruchung in Bezug auf Arbeitsintensität und Länge der Arbeitszeit im Zusammenhang mit Büroarbeit. Insbesondere die Gefahr der informationellen Überforderung ist hinreichend belegt. So zeigten z. B. Untersuchungen zur E-Mail-Kommunikation einen Anstieg erlebter Überforderung in Abhängigkeit von der Anzahl eingehender Meldungen je Zeiteinheit, vom Inhalt, von der Darstellung sowie von der beim Eingang der Meldung vorliegenden Arbeitssituation (vgl. Moser et al. 2002; Simpson und Prusak 1995). Unübersichtliche Informationsdarstellungen oder unerwartetes Systemverhalten können den ,,Technikstress“ weiter ansteigen lassen (Hoppe 2010). Mit zunehmendem Einsatz digitaler Technologien in der wissenschaftlichen Arbeit und Kommunikation lässt sich auch eine Zunahme solcher Fehlbeanspruchungen bei wissenschaftlich Beschäftigten annehmen.

Arlinghaus (2017) stellte eine Übersicht gesicherter arbeitswissenschaftlicher Erkenntnisse zur Belastung und Beanspruchung von Wissensarbeitenden zusammen. Unter anderem werden hier negative gesundheitliche und soziale Auswirkungen der durch die Digitalisierung geförderten ständigen Erreichbarkeit aufgeführt. Weiterhin ist die Arbeit im Home-Office häufig mit einer sozialen Isolation verbunden und dem Wegfall von Pausen, jedoch zeigen Studien auch eine subjektiv erhöhte Leistungsfähigkeit und eine bessere Vereinbarkeit von Aufgaben des Berufs- und Privatlebens. Unregelmäßige und lange tägliche und wöchentliche Arbeitszeiten sowie zu kurze Ruhezeiten zeigen wiederum zahlreiche gesundheitliche Auswirkungen, ein erhöhtes Unfallrisiko und verringerte Leistung sowie eine Verschlechterung der Work-Life-Balance.

In ihrer Arbeit sind Wissensarbeitende zwar häufig konfrontiert mit hohen (meist kognitiven) Arbeitsanforderungen, gleichzeitig sind sie jedoch auch zumeist in der Lage, über Art und Inhalt ihrer Arbeitstätigkeiten maßgeblich mitzubestimmen. Ihre Arbeit gewährt ihnen folglich einen hohen Handlungsspielraum, was häufig mit einer größeren Arbeitszufriedenheit einhergeht. Selbstbestimmte Arbeitszeiten wurden auch mit positiven Effekten auf die Gesundheit, Work-Life Balance und die soziale Teilhabe assoziiert (Arlinghaus 2017).

Es ist anzunehmen, dass über die o. g. Aspekte der Wissensarbeit auch wissenschaftliche Arbeit von einer erhöhten zeitlichen und örtlichen Flexibilisierung durch die Digitalisierung profitieren könnte. Die mit der Digitalisierung oftmals einhergehende Automatisierung reduziert in vielen Bereichen (physische und kognitive) Routinetätigkeiten, während der Anteil an nicht-routinierten, insbesondere komplexen, Tätigkeiten zunimmt. Dies schafft Bedingungen, die der wissenschaftlichen Arbeit, welche sich in der Regel mit komplexen Sachverhalten auseinandersetzt und 
die häufig nach kreativen und innovativen Einfällen verlangt, welche oftmals auch außerhalb regulärer Büroarbeitszeiten entstehen, entgegenkommen könnten. Es liegt daher nahe, dass Forschende vom Einsatz digitaler Technologien mehr profitieren könnten als andere Berufsgruppen. Forschende haben außerdem in der Regel hohe Freiheitsgrade in Bezug auf Arbeitsinhalte, was mit einer erhöhten intrinsischen Arbeitsmotivation einhergeht. Es ist daher zu vermuten, dass Forschende, aufgrund eines höheren Maßes an Selbstbestimmtheit und intrinsischer Arbeitsmotivation resilienter gegenüber digitalen Stressoren sind als andere Berufsgruppen.

Die durch die Digitalisierung vorangetriebene Entgrenzung der Arbeit geht häufig mit einer Verlagerung in risikoreiche Arbeitsrechtsverhältnisse einher, wie Hacker (2018) in seiner Diskussion zur innovativen Erwerbsarbeit darstellt. Durch den Mangel an Zeit- oder Leistungsnormen in diesem Bereich erhöht sich außerdem die Gefahr einer kontinuierlichen Überforderung: Eine hohe Flexibilisierung der Arbeit in Kombination mit einer bei wissenschaftlich Beschäftigten häufig vorliegenden hohen intrinsischen Arbeitsmotivation erhöht damit die Wahrscheinlichkeit, dass Arbeitspersonen Arbeits- und Pausenzeiten wählen, die nicht nach ergonomischen Maßstäben und damit wenig menschenverträglich gestaltet sind. Da Wissenschaftler/ Wissenschaftlerinnen an universitären und außeruniversitären Forschungseinrichtungen in der Regel heute bereits in zeitlich befristeten Arbeitsverhältnissen mit unklarer Berufsperspektive arbeiten, in Befragungen zumeist eine erhebliche Anzahl geleisteter (unbezahlter) Überstunden sowie eine deutliche Entgrenzung von Berufsund Privatleben angeben (z. B. Müller 2015; Eichhorst 2015), ist noch nicht abzusehen, ob sich die Arbeitsbedingungen für Beschäftigte in der Wissenschaft mit der Digitalisierung maßgeblich verändern würden. Wahrscheinlicher erscheint es, dass das in der Politik bereits erkannte und häufig im gesellschaftlichen Diskurs thematisierte Problem prekärer Beschäftigungsverhältnisse von wissenschaftlich Arbeitenden zu einer positiven Entwicklung für diese Berufsgruppe führt. Um den gesundheitlichen Nebenwirkungen der Digitalisierung entgegen $\mathrm{zu}$ wirken, bedarf es hingegen noch weiterführender Maßnahmen zur gesellschaftlichen Sensibilisierung und Aufklärung in Bezug auf eine menschenverträgliche Arbeitsgestaltung.

\subsection{KI und Automation der wissenschaftlichen Arbeit}

Betrachtet man einige der in vielen Bereichen als ,,intelligent“ bezeichneten Technologien genauer, lässt sich feststellen, dass diese heutzutage in erster Linie für die Automatisierung von Arbeit eingesetzt werden, also für die technikbetriebene Durchführung von Arbeitsprozessen, die zuvor vollständig oder teilweise von menschlichen Arbeitskräften durchgeführt wurden (z. B. Hirsch-Kreinsen und Karačić 2019). Das Phänomen der Automatisierung von Arbeit wurde in den vergangenen Jahrzehnten in der Arbeitsforschung, Ingenieurpsychologie und weiteren verwandten Fachgebieten ausgiebig erforscht. Während in der Arbeitsforschung insbesondere die Erforschung und Ermittlung des Substituierbarkeitspotenzials von 
Automationstechnologien und dessen Auswirkungen auf den Arbeitsmarkt von Interesse ist, steht in der Ingenieurpsychologie und Human Factors Forschung die Untersuchung von Wirkmechanismen auf der Ebene des Individuums im Fokus. Es ist davon auszugehen, dass der Einsatz von KI-Technologien auch die Automatisierung der wissenschaftlichen Arbeit weiter vorantreibt. Im folgenden Abschnitt sollen daher näher auf absehbare und mögliche Folgen der Automation für die wissenschaftliche Arbeit eingegangen werden.

Studien zur Schätzung des Substituierbarkeitspotenzials von Automationstechnologien stützen sich in der Regel auf dem in Tätigkeitsprofilen oder durch Selbstauskünfte beschriebenen Anteil von Routinetätigkeiten, also regelmäßig wiederkehrenden Vorgängen eines Berufs. Je größer der Anteil dieser Tätigkeiten, desto größer wird die Wahrscheinlichkeit eingeschätzt, dass die Arbeit durch Technologien ausgeführt werden kann. Die grundlegende Annahme ist hier, dass Arbeitsaufgaben automatisiert werden können, wenn sich diese in Gänze durch eine Computer-Anwendung beschreiben und ausführen lassen. So kam z. B. eine Untersuchung des Zentrums für Europäische Wirtschaftsforschung (ZEW) zu dem Schluss, dass neue Technologien nicht nur fähig seien, manuelle Routinearbeit zu ersetzen, sondern vor allem auch Arbeit mit einem hohen Anteil geistiger Routinearbeit zunehmend substituiert wird (vgl. Arntz et al. (2019)).

Diese Verschiebung der Anteile, die Menschen und Software-Lösungen bei der Erfüllung einer Aufgabe haben, wurde von der SAE International für das Thema „Autonomes Fahren“ beschrieben (SAE International 2016). Das entsprechende Phasenmodell ist in Abb. 5.1 dargestellt.

Das Beispiel des autonomen Fahrens ist gut geeignet, um diese Veränderungen darzustellen. Während ursprünglich die Fahrer alle Aktionen, wie Lenken, Bremsen, Gas geben etc., selbstständig ausgeführt haben, werden zukünftig immer mehr Aufgaben von Software-Lösungen übernommen, wobei nicht zwingend KIAlgorithmen eingesetzt werden müssen (Buxmann 2020). Das hier dargestellt Grundprinzip lässt sich auf eine Vielzahl anderer Bereiche aus Wissenschaft und Wirtschaft übertragen. Mit dem Einsatz von KI-Anwendungen können Effizienz-

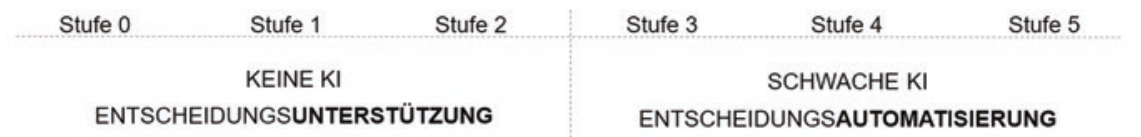

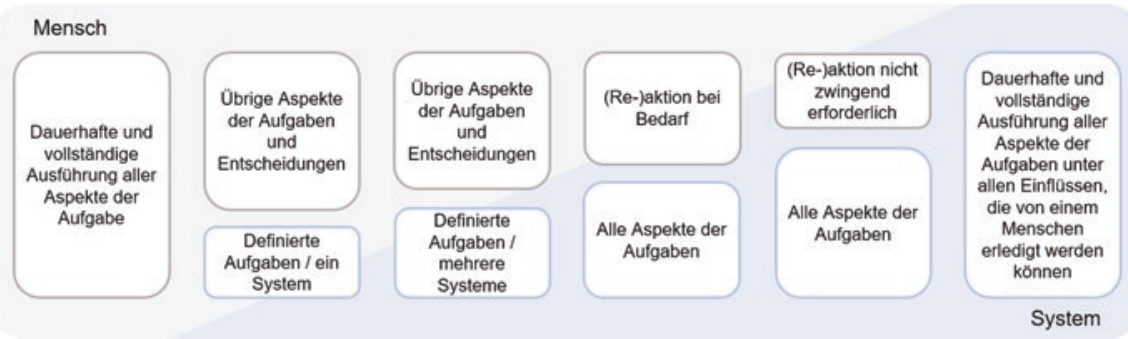

Abb. 5.1 Arbeitsteilung zwischen Mensch und Maschine in Anlehnung an das Phasenmodell der SAE International (verändert nach SAE International 2016) 
gewinne verbunden sein, darüber hinaus ist es aber auch denkbar, dass KI-Anwendungen bessere Entscheidungen als Menschen treffen. So könnten KIAnwendungen einen immer größeren Teil der Literaturrecherche übernehmen oder bei Formulierung von Standardtexten helfen. Neben den möglichen Effizienz- und Effektivitätseffekten ist aber auch zu berücksichtigen, dass ein höherer Anteil von Arbeiten, die von KI-Anwendungen übernommen werden, Verantwortlichkeiten für Entscheidungen vom Menschen weg hin zu KI-Anwendungen verschieben. Vor diesem Hintergrund ist eine breite gesellschaftliche Debatte notwendig wieviel Verantwortung zukünftig bei KI-Anwendungen liegen sollte.

Auf makroökonomischer Ebene wurden in einer 2018 vom World Economic Forum durchgeführten Studie „The Future of Jobs 2018“ über 300 Unternehmen nach den Auswirkungen der Digitalisierung auf die Zukunft der Arbeit gefragt. In Abb. 5.2 ist dargestellt, welche Verschiebungen zwischen Mensch und Maschine bis 2022 stattfinden könnten.

\section{DURCHSCHNITT (ÜBER 12 BRANCHEN)}

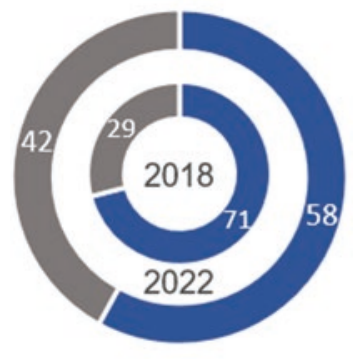

- Mensch = Maschine

\section{BEGRÜNDEN/} ENTSCHEIDEN

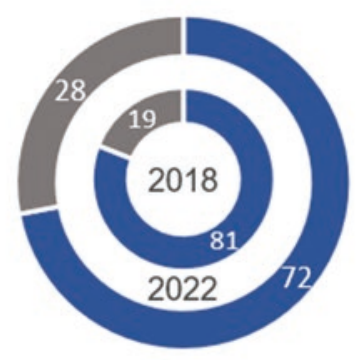

- Mensch " Maschine
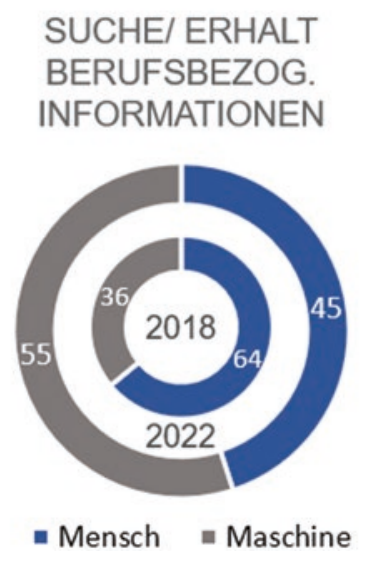

- Mensch $=$ Maschine

\section{VERWALTUNG}

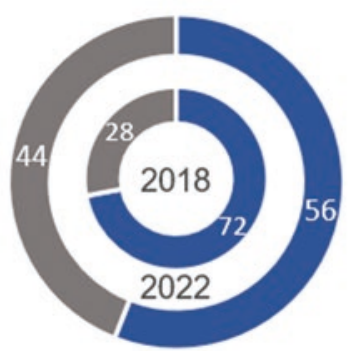

- Mensch = Maschine

\section{KOMPLEXE + TECHNISCHE TÄTIGKEITEN}

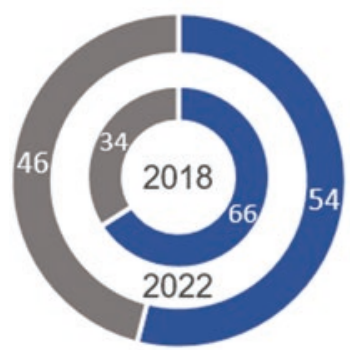

- Mensch = Maschine

\section{INFORMIEREN/ \\ DATEN \\ VERARBEITEN}

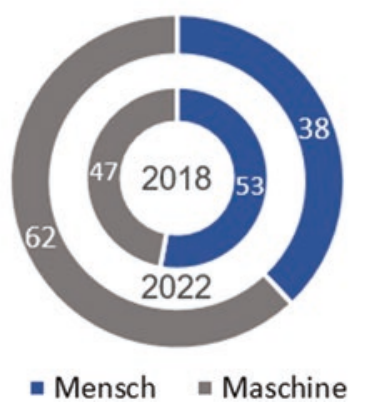

Abb. 5.2 Maschinen übernehmen die Arbeit - Aufteilung der Arbeitsstunden zwischen 2018 und 2022/Anteile in Prozent (verändert nach World Economic Forum 2018 und Schmidt 2018) 
Ein zentrales Resultat der Studie ist, dass KI-Anwendungen zukünftig viele Aufgaben übernehmen werden, die bislang von Menschen durchgeführt wurden. Ein weiteres wichtiges Ergebnis ist, dass KI immer stärker auch Aufgaben übernehmen könnte, für die kognitive Fähigkeiten erforderlich sind. Das bedeutet beispielsweise, dass zukünftig Menschen auch bei höherwertigen Aufgaben, wie Argumentation und Entscheidungsfindung, durch KI-Anwendungen unterstützt werden. Das könnte auch für die Arbeit von Wissenschaftlern/Wissenschaftlerinnen gelten, etwa im Bereich der Medizin bei der Diagnose und Therapie von Krankheiten. Hierbei ist anzumerken, dass in der Informatik zurzeit weltweit viele Arbeiten rund um das Thema „Explainable Artificial Intelligence“ durchgeführt werden (Adadi und Berrada 2018). Dies kann zukünftig helfen, Entscheidungen der KI besser zu erklären, was für die Akzeptanz der Lösung, beispielsweise für Ärzte, essenziell ist.

Vor diesem Hintergrund kommen viele Studien zu dem Ergebnis, dass KI zu einer Vernichtung von Arbeitsplätzen führen wird. So zeigt eine viel zitierte Studie der Oxford Universität, dass bis zu 47 Prozent aller US-amerikanischen Jobs stark von KI gefährdet seien (Frey und Osborne 2017). In diesem Zusammenhang warnt z. B. die OECD vor einem umfangreichen Stellenabbau. Rund 14 Prozent der Jobs in den Mitgliedsstaaten, darunter auch Deutschland, seien hochgradig automatisierbar, und 32 Prozent der Jobprofile könnten sich stark verändern (OECD 2018). Während diese Studien die Auswirkungen der KI auf den Arbeitsmarkt aufgabenbasiert erfassen, hat der Stanford-Forscher Michael Webb eine neue Methode entwickelt (Webb 2019): Er hat die Beschreibungen der aktuellen Tätigkeiten der Beschäftigten mit den Beschreibungen der Patentanmeldungen zur KI verglichen. Die Überlappungen zeigen das Substitutionspotenzial. Steht zum Beispiel in der Aufgabenbeschreibung eines Arztes „Diagnose des Patientenzustandes“, hat Webb die Patentanmeldungen mit dem Begriff „Zustandsdiagnose“ oder ähnlichen Wörtern gesucht. Die Häufigkeit der gefundenen Patente gibt Aufschluss über die Wahrscheinlichkeit einer KI-induzierten Automatisierung dieser Tätigkeit. Die Ergebnisse decken sich zu großen Teilen mit denen der aufgabenorientierten Studien, jedoch konnte er auch zeigen, dass von der KI innovative Tätigkeiten bzw. solche die Neuland betreten (zum Beispiel neue wissenschaftliche Projekte) weniger stark betroffen sind. (Webb 2019; Buxmann und Schmidt 2020).

Diese Angaben zu Ersetzungspotenzialen sind jedoch stets mit Vorsicht zu interpretieren, denn sie treffen notwendigerweise zahlreiche Annahmen, u. a. zu getätigten Investitionen in die Digitalisierung von Unternehmen, zur Beurteilung der Routinehaftigkeit von Berufen und zur Entwicklung der Fähigkeiten von Technologien. Zu beachten ist dabei ferner, dass das Substituierbarkeitspotenzial nicht zwangsläufig zu einer höheren Arbeitslosigkeit führt, sondern lediglich - zumindest auf Bundesebene - Veränderungen in der Struktur der Beschäftigung zu beobachten sein werden. So werden z. B. größere Zuwächse an Arbeitsplätzen in den Bereichen der geistes- und sozialwissenschaftlichen und künstlerischen Berufe und der Berufe in IT und Naturwissenschaften erwartet (vgl. Zika et al. 2018).

Speziell in Bezug auf die kostenabhängige Realisierung von Wissensarbeit merkte Hacker (2018) an, dass es auch zukünftig noch längere Zeit Nischen geben wird, in denen menschliche Wissensarbeit günstiger als digitale Lösungen ist. Jedoch gab er mit Blick auf die historische Entwicklung des globalen Arbeitsmarkts auch zu be- 
denken, dass damit auch die verbleibenden Arbeitsplätze vermutlich in Länder abwandern würden, in denen diese Arbeit günstiger verrichtet werden könnte. Bereits heute befeuern neue, durch die Digitalisierung ermöglichte Formen der Arbeit, wie z. B. das Crowdsourcing, vor dem Hintergrund der Plattformökonomie den globalen Wettbewerb unter hochqualifizierten Arbeitskräften. Diese neuen Arbeitsformen gehen nicht selten auch mit prekären Beschäftigungsverhältnissen einher.

Doch es werden auch technologische Grenzen der Substituierbarkeit (zumindest in der näheren Zukunft) gesehen. In ihrer weltweit viel zitierten Studie zur Schätzung des Substituierbarkeitspotenzials in den USA gingen Frey und Osborne (2013) davon aus, dass drei Aspekte menschlicher Fähig- und Fertigkeiten in naher Zukunft nicht von Technologien ersetzt werden können, die sie unter den Begriffen Wahrnehmung und Feinmotorik, soziale Intelligenz und kreative Intelligenz zusammenfassten. Hacker (2018) sah ebenfalls eine Automatisierungsgrenze bei der kreativen Innovationsarbeit. Vor dem Hintergrund kognitionspsychologischer Überlegungen spezifizierte er, dass geistige Arbeit nicht automatisiert werden könnte, wenn diese die Suche nach Lösungen beinhaltet, bei denen weder Ziel noch der Weg zur Zielerreichung vorgegeben ist. Hingegen kann der Mensch dort ersetzt werden, ,wo eindeutige Verarbeitungsregeln der Information (Algorithmen) vorliegen und prinzipiell auch im Übergangsfeld, wo definierbare Findehilfen (Heuristiken) angegeben werden können.“ (Hacker 2018, S. 9).

Selten ersetzen Technologien ganze Berufe, viel häufiger werden an Arbeitsplätzen nur einzelne Tätigkeitsbereiche automatisiert. Die Einführung automatisierter Systeme an Arbeitsplätzen bewirkt damit nicht nur eine Veränderung von Arbeitstätigkeiten der dort eingesetzten Menschen (vgl. Autor und Price 2013), sondern birgt auch Risiken, die es bei der Gestaltung dieser Arbeitsplätze und der Schulung von Personal zu berücksichtigen gilt. Diese Risiken liegen hauptsächlich in einer aus arbeitswissenschaftlichen Sicht unangemessenen Umsetzung des Automationsgrads im Falle einer Teilautomatisierung. Es ist davon auszugehen, dass diese auch teil-automatisierte wissenschaftliche Arbeit betreffen, insbesondere die Datenakquise und -verarbeitung.

Mit höheren Automationsgraden fällt es dem Menschen zunehmend schwerer, die agierenden Mechanismen und den Zustand des Systems nachzuvollziehen. Als Folge sinken die Prozesstransparenz und die Fähigkeit des Menschen, Fehler in den Handlungen oder den Handlungen zugrunde liegenden Annahmen zu detektieren oder gar zu korrigieren. Soll der Mensch bei teilautomatisierten Arbeitsaufgaben nicht nur die Entscheidungshoheit, sondern auch die Entscheidungskompetenz behalten, ist daher auf die Umsetzung einer menschzentrierten Automation zu achten. Diese erfordert u. a. den Einsatz geeigneter Benutzungsschnittstellen und eine lernförderliche Arbeitsgestaltung.

Zusammenfassend lässt sich an dieser Stelle festhalten, dass auch wissenschaftliche Arbeit von der durch KI und anderen digitalen Technologien vorangetriebenen Automatisierung betroffen sein wird. Zum einen wird sie Arbeitstätigkeiten verändern und damit auch zweifellos eine Entlastung von ungeliebten und nicht wertschöpfenden Routinetätigkeiten (insbesondere im administrativen Bereich) bringen. Auf der anderen Seite wird sie jedoch auch den Arbeitsexport und globalen Wettbewerbsdruck in der Wissenschaft befördern. Bei der Einführung von neuen Auto- 
mationstechnologien sollten Anwendende weiterhin für einen angemessenen Umgang mit diesen Technologien sensibilisiert und ein grundsätzliches Verständnis der Mechanismen und der Limitationen der Technologien geschafft werden.

\subsection{Empirische Befunde zu den Auswirkungen von KI und Digitalisierung auf die Arbeit und Arbeitsbedingungen von Forschenden}

Eine im Jahr 2019 im Auftrag des IQIB-Instituts für qualifizierende Innovationsforschung und -beratung $\mathrm{GmbH}$ durchgeführte Umfrage lieferte erste Anhaltspunkte für die Bewertung möglicher Auswirkungen von Digitalisierung im Allgemeinen und KI-basierten Technologien im Speziellen auf die Arbeit und Arbeitsbedingungen von Forschenden (Rick et al. 2021). ${ }^{1}$

Befragt wurden 166 Wissenschaftlerinnen und Wissenschaftler im Raum Nordrhein-Westfalen (s. Abb. 5.3 zur Beschreibung der Stichprobe), die zum Zeitpunkt der Befragung an Universitäten und Fachhochschulen, sowie außeruniversitären Forschungseinrichtungen (DLR, DFKI, verschiedene Fraunhofer Einrichtungen) beschäftigt waren. Die Teilnehmenden ordneten sich den Fachdisziplinen der Naturwissenschaften (15\%), der Ingenieurwissenschaft und Informatik (62\%) oder der Geistes- und Sozialwissenschaft (23\%) zu.

\subsubsection{Auswirkungen der Digitalisierung auf den Forschungsalltag}

Die Umfrage bestätigte, dass wesentliche Merkmale der Wissensarbeit auch auf die wissenschaftliche Arbeit zutrafen. So zeigte sich hier unter anderem, dass auch die wissenschaftliche Arbeit sehr kommunikationsintensiv ist und hierfür sehr häufig

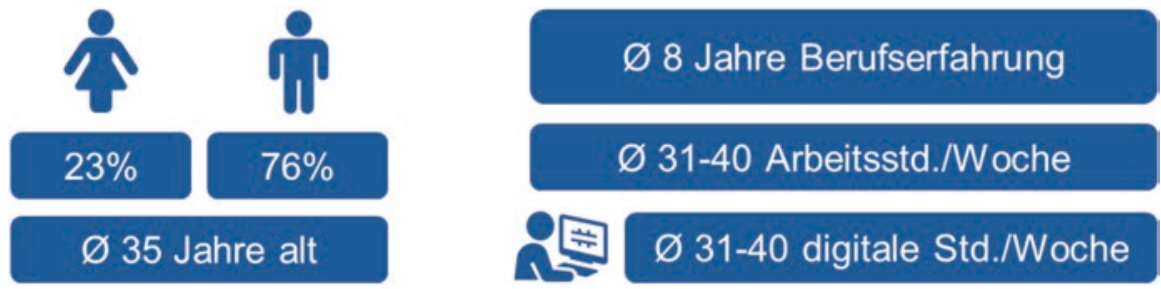

Abb. 5.3 Beschreibung der Stichprobe (eigene Darstellung)

\footnotetext{
${ }^{1}$ Die Begleitstudie wurde von Vera Rick (RWTH Aachen), Julia Distelrath (IQIB) und Verena Nitsch (RWTH Aachen) durchgeführt. Detailliertere Ausführungen zur Studie und den Ergebnissen wurden auf dem Publikationsserver der RWTH Aachen University veröffentlicht und sind frei zugänglich unter https://publications.rwth-aachen.de/record/811477/files/811477.pdf.
} 
digitale Technologien genutzt wurden. Für das kollaborative wissenschaftliche Arbeiten wurden jedoch in erster Linie Technologien eingesetzt, mit denen man nur gelegentlich Kontakt zu anderen Personen hat, z. B. Videokonferenzdienste, oder mit denen Interaktionen eher indirekt vollzogen werden, z. B. über webbasierte Dienste für den Datenaustausch und die Wissensdokumentation. Weniger verbreitet hingegen waren Technologien zur direkten kollaborativen Arbeit, wie z. B. kollaborative Schreibprogramme und Projektmanagement-Software (s. Abb. 5.4).

Überraschenderweise scheint mobiles Arbeiten unter den befragten Wissenschaftlerinnen und Wissenschaftlern nicht so weit verbreitet zu sein, wie häufig angenommen. So gaben zwar rund zwei Drittel (68 \%) der Befragten an, bei ihrer derzeitigen Tätigkeit Telearbeit, Homeoffice oder mobiles Arbeiten zu nutzen, von diesen Personen nahm jedoch nur ein Drittel (33\%) das Angebot ein- oder mehrmals pro Woche in Anspruch, während rund die Hälfte (55\%) dies nur einmal pro Monat oder seltener taten (s. Abb. 5.5). Es ist dabei jedoch anzumerken, dass die Umfrage vor der Covid-19 Pandemie stattfand. Aufgrund der pandemiebedingten Restriktionen haben sich in vielen deutschen Unternehmen technische, organisatorische und personelle Rahmenbedingungen zugunsten der Homeoffice-Arbeit entwickelt. Es lässt sich vermuten, dass diese Entwicklungen auch an vielen Forschungsstandorten stattfanden und damit das Homeoffice zukünftig auch mehr für wissenschaftliche Arbeit genutzt wird. Ob diese Umstellung nur von kurzer oder längerer Dauer ist, lässt sich zum Zeitpunkt dieser Veröffentlichung noch nicht abzuschätzen.

Obgleich zum Zeitpunkt der Befragung vergleichsweise wenig Personen häufig mobil arbeiteten, äußerten rund zwei Drittel der Befragten (67\%), dass der zunehmende Einsatz von digitalen Technologien die Grenzen zwischen Arbeits- und Privatleben auflöst. Wenige Personen verbanden damit jedoch ein erhöhtes Konfliktpotenzial oder Schwierigkeiten bei der Erledigung privater Aufgaben (s. Abb. 5.6).

Rund die Hälfte der befragten Wissenschaftlerinnen und Wissenschaftler schrieben dem Einsatz digitaler Technologien eine Vergrößerung ihres Handlungsspielraums zu. So gaben $48 \%$ der Befragten an, dass digitale Technologien eine freiere

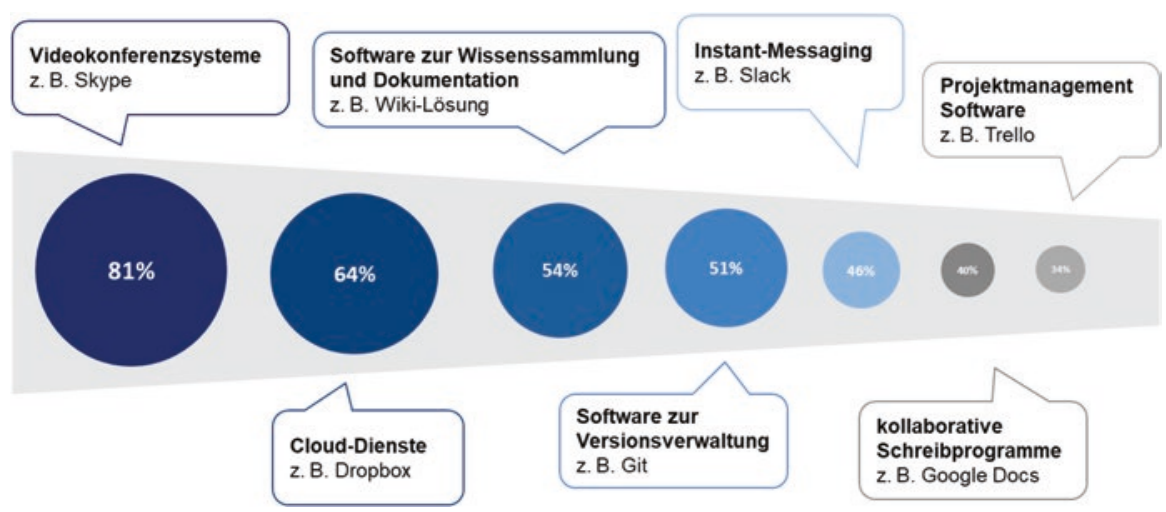

Abb. 5.4 Verwendete Technologien zur Unterstützung der kollaborativen wissenschaftlichen Arbeit (eigene Darstellung) 
Nutzen Sie bei Ihrer derzeitigen Tätigkeit Telearbeit, Homeoffice oder mobiles Arbeiten?

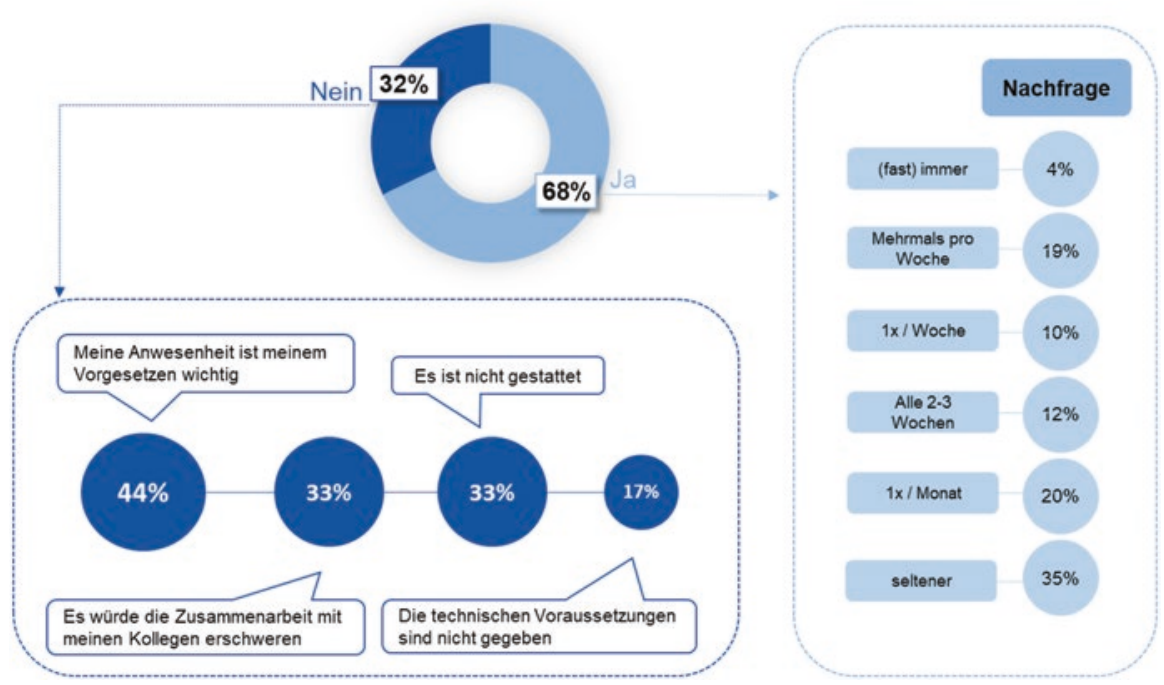

Abb. 5.5 Angaben zur Nutzung von Möglichkeiten des mobilen Arbeitens (eigene Darstellung)

\section{Verwischung der Grenze zwischen Arbeits- und Privatleben durch digitale Technologien.}

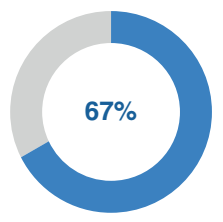

Digitale Technologien führen zu ...

Konflikten mit dem Privatleben.

$24 \%$

Problemen bei der Fertigstellung privater Aufgaben

$19 \%$

Abb. 5.6 Angaben zur technologiegetriebenen Entgrenzung von Arbeit (eigene Darstellung)

zeitliche Einteilung der Arbeit ermöglichen und $46 \%$ stimmten der Aussage zu, dass digitale Technologien eine größere Entscheidungsautonomie geben.

Auch in Bezug auf Produktivität und Qualität der Arbeit wird der Einfluss von digitalen Technologien im Forschungsalltag positiv gesehen. So sahen z. B. $58 \%$ der Befragten eine Verbesserung der Produktivität und ebenso viele eine qualitative Verbesserung der Arbeitsergebnisse durch den Einsatz von digitalen Technologien. Nur rund ein Viertel der befragten Personen waren der Ansicht, dass digitale Technologien den Zeitdruck bei der Arbeit erhöhten (Abb. 5.7). Fast alle (92\%) fühlten sich nach eigenen Angaben hinreichend kompetent, um die Potenziale digitaler Unterstützung zu nutzen. Weiterbildung im Umgang mit digitalen Technologien erfolgte bei den meisten Personen (91\%) in Eigenregie durch Ausprobieren; nur $15 \%$ gaben an, dafür Schulungen und Weiterbildungen des Arbeitgebers in Anspruch zu nehmen. 
Digitale Technologien führen in meinem Alltag zu...

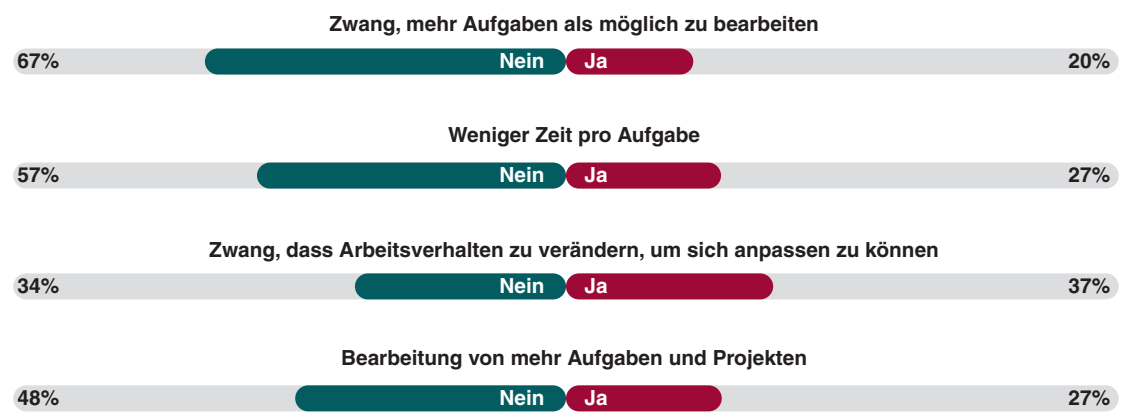

Abb. 5.7 Auswirkung digitaler Technologien auf den subjektiven Zeitdruck (eigene Darstellung)

\subsubsection{Angaben zum Einsatz von KI-basierten Technologien}

Zunächst wurden die Befragten gebeten, zu einer Vielzahl von Technologien die Einschätzung abzugeben, ob es sich ihrer Meinung nach dabei um eine KITechnologie handelt. Das Ergebnis der Befragung ist in Abb. 5.8 dargestellt. Die Antworten deuten darauf hin, dass auch bei hochqualifizierten Personen mit selbsteingeschätzter hoher Kompetenz im Umgang mit digitalen Technologien eine Unklarheit darüber zu herrschen scheint, was tatsächlich als „KI“ bezeichnet werden kann. So wurden z. B. Technologien wie Data Mining Tools und Suchmaschinen von der Mehrheit der Befragten nicht als KI-Technologie eingestuft. Die Begriffe Mustererkennung, maschinelles Lernen und Deep Learning hingegen wurden zwar von einer Mehrheit der Kategorie „KI“ zugeordnet, jedoch war auch hier jede 5. Person einer anderen Meinung (s. Abb. 5.8).

Um für die weitere Befragung eine gemeinsame Verständnisgrundlage zu schaffen, wurde anschließend KI folgendermaßen definiert:

„Künstliche Intelligenz (KI) beschreibt die Fähigkeit einer Computer-Anwendung, Eigenschaften menschlicher Intelligenz, aufzuweisen, beispielsweise durch hören, sehen, lernen, sprechen, planen oder schlussfolgern. Demnach sind beispielsweise auf Programme zur Gesichtserkennung als KI-basierte Technologie einzustufen, da Eigenschaften wie sehen und lernen vorhanden sind. "

Dieser Definition folgend gaben nur rund ein Viertel der Befragten an, nicht mit KI Technologien zu arbeiten, allerdings wurden diese von den Befragten vergleichsweise wenig ( $<10$ Std./Woche) eingesetzt, insbesondere von Geistes- und Sozialwissenschaftlerinnen und -wissenschaftlern.

Eine differenzierte Betrachtung der Einstellungen von Wissenschaftlern/Wissenschaftlerinnen gegenüber KI-Technologien in Abhängigkeit von der selbstbenannten Nutzung solcher Technologien zeigt einige Unterschiede auf. So stimmten Personen, die im Rahmen ihrer Arbeit nach eigenen Angaben viel Gebrauch von KI-Technologien machten ( $\geq 21$ Std./Woche), der Aussage, dass KI die wissen- 


\begin{tabular}{|c|c|c|c|}
\hline \multicolumn{2}{|l|}{ Keine KI } & \multicolumn{2}{|l|}{$\mathbf{K I}$} \\
\hline PCs & $100 \%$ & Maschinelles Lernen & $82 \%$ \\
\hline Videokonferenzdienste & $100 \%$ & Deep Learning & $81 \%$ \\
\hline Textverarbeitungsprogramme & $\mathbf{9 9} \%$ & Mustererkennung & $80 \%$ \\
\hline Webbasierte Kommunikation & $99 \%$ & Problemlösung & $76 \%$ \\
\hline Fernzugang/VPN & $\mathbf{9 9} \%$ & Übersetzungsprogramme & $65 \%$ \\
\hline Smartphones & $97 \%$ & Digitale Assistenten & $64 \%$ \\
\hline Bildverarbeitung & $\mathbf{9 5} \%$ & Bilderkennung & $59 \%$ \\
\hline Hochleistungsrechensysteme & $95 \%$ & Chatbots & $\mathbf{5 0 \%}$ \\
\hline Literatursuchprogramme & $\mathbf{9 0} \%$ & Big Data & $33 \%$ \\
\hline Suchmaschinen & $65 \%$ & & \\
\hline Data Mining Tools & $62 \%$ & & \\
\hline
\end{tabular}

Abb. 5.8 KI-Klassifikation von verschiedenen Technologien nach Einschätzung der Befragten (eigene Darstellung)

schaftliche Selbstbestimmtheit oder die Standards guter wissenschaftlicher Praxis beeinträchtigt deutlich seltener zu als Personen, die weniger mit derartigen Technologien arbeiteten (s. Abb. 5.9). Hierbei ist jedoch auch zu beachten, dass sich die Größe der jeweiligen Stichproben auch stark unterscheidet, so dass ein Vergleich der relativen Häufigkeiten der Aussagen nur bedingt aussagekräftig ist.

Unabhängig von der KI-Nutzung zeigten die befragten Wissenschaftlerinnen und Wissenschaftler jedoch eine überwiegend positive Meinung von den Auswirkungen dieser Technologien auf den eigenen Forschungsalltag. Einzig in Bezug auf den durch die Technologien zu erwartenden Fortschritt zeigten sich hier größere Unterschiede zwischen den beiden Nutzergruppen (s. Abb. 5.10).

Doch den Befragten waren auch Limitationen der Technologien weitgehend bekannt. So war weniger als ein Viertel der Befragten (23\%) der Ansicht, dass KI-Technologien objektive Ergebnisse liefern. Immerhin $39 \%$ der Befragten waren der Ansicht, dass damit Ergebnisse erzeugt werden, die von Ihnen nicht unmittelbar nachvollzogen werden können.

Mit offenen Fragen wurde nach absehbaren Folgen von KI-Technologien sowie nach Wünschen und Befürchtungen gefragt, die von etwas weniger als der Hälfte der Befragten beantwortet wurden. In Bezug auf absehbare Folgen sahen lediglich $5 \%$ dieser Personen eine Zunahme der Bedeutung der Informatik in der Wissenschaft und $11 \%$ eine Veränderung ihres Themenschwerpunkts. Die Mehrheit der 


\section{KI beeinträchtigt die wissenschaftliche Selbstbestimmtheit}

\section{KI-Nutzung $>=21$ \\ Std./Woche \\ $(\mathrm{N}=21)$}

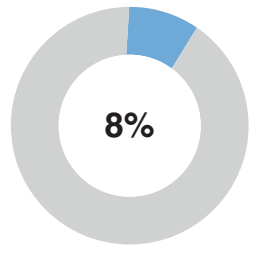

KI beeinträchtigt die
Standards guter
wissenschaftlicher Praxis

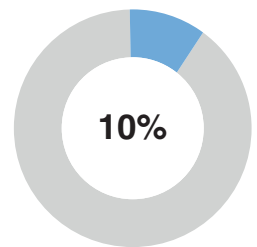
KI-Nutzung $<21$
Std./Woche
$(\mathrm{N}=145)$
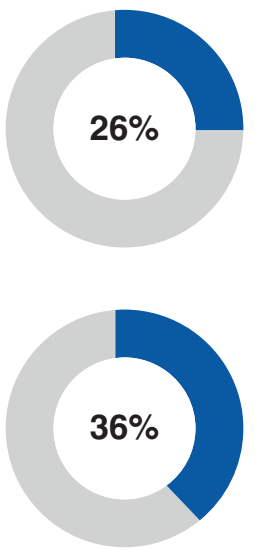

Abb. 5.9 Eingeschätzte Beeinträchtigungen des wissenschaftlichen Arbeitens in Abhängigkeit der Nutzung von KI-basierten Technologien (\% der Teilnehmenden, die die jeweilige Aussage mit „trifft zu“ und „trifft voll und ganz zu“ bewerteten) (eigene Darstellung)

KI Technologien...

bieten Unterstützung in der Forschung

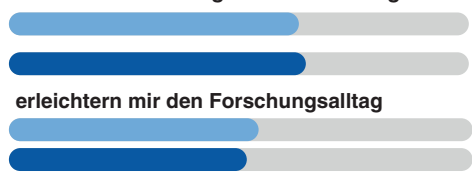

erleichtern mir die wissenschaftliche Arbeit

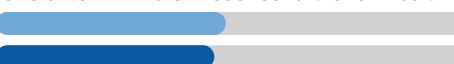

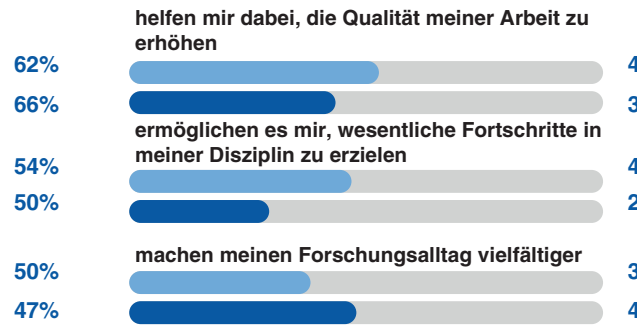

$48 \%$

$39 \%$

$43 \%$

$24 \%$

$37 \%$

$45 \%$

Abb. 5.10 Bewertung des Einflusses von KI Technologien auf den Forschungsalltag (hellblau: mindestens 21 Std./Woche KI-Nutzung; dunkelblau: höchstens 20 Std./Woche KI-Nutzung) (eigene Darstellung)

Befragten erhoffte sich jedoch Prozessoptimierungen durch KI-Technologien. Nur $3 \%$ der Befragten waren der Ansicht, dass KI keine absehbaren Folgen haben würde. Lediglich $8 \%$ der Befragten sahen einen Stellenabbau in der Wissenschaft voraus. Weitere Befürchtungen sind in Abb. 5.11 zusammengefasst dargestellt.

Wünsche an KI-Technologien wurden insbesondere in Bezug auf Nutzerfreundlichkeit (gemeinsame Standards, Transparenz, Zuverlässigkeit) geäußert (45\%), aber auch die Übernahme von Routinetätigkeiten hatten sich einige Personen (27\%) erhofft. Die Sicherstellung von Datensicherheit, Privacy und des ethisch vertretbaren Einsatzes wurde von $12 \%$ der Befragten gefordert. 


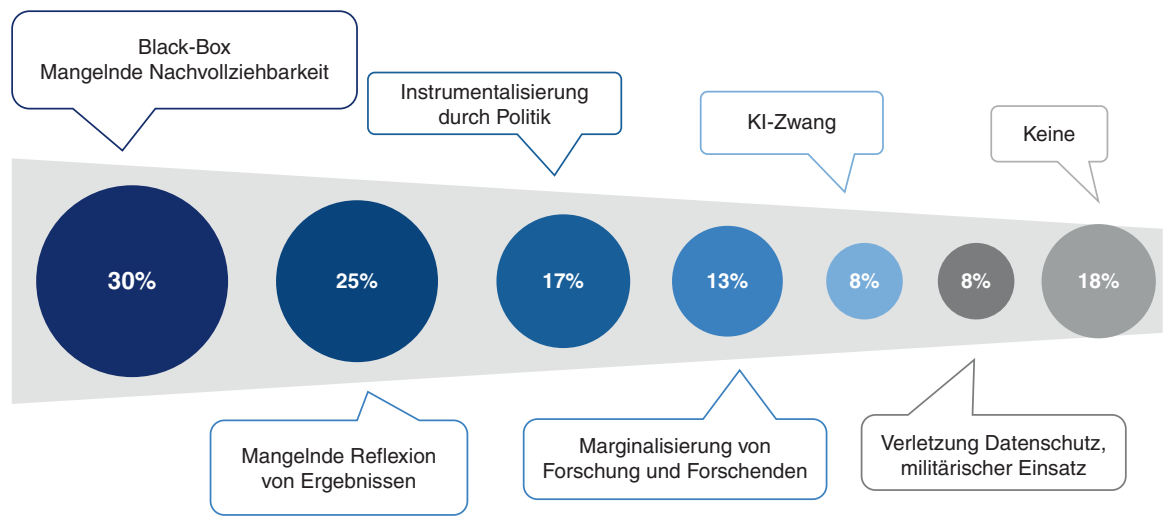

Abb. 5.11 Befürchtungen der befragten Wissenschaftlerinnen und Wissenschaftler gegenüber KI-Technologien (eigene Darstellung)

Die Ergebnisse der Umfrage sollten zweifellos mit Vorsicht interpretiert werden. Zum einen bilden sie nur eine Momentaufnahme ab, die aufgrund dynamischer technologischer, organisatorischer und kultureller Entwicklungen in Unternehmen eine kurze Halbwertzeit besitzt. Sowohl die Nutzung als auch die subjektive Bewertung von neuen Technologien werden stark von der „Expositionsdauer“ als auch einzelnen, bemerkenswerten Ereignissen beeinflusst (z. B. der Berichterstattung von verunfallten automatisierten Fahrzeugen oder populären Filmen). Zum anderen lassen sich aus der sehr selektiven Stichprobe von Befragten kaum Aussagen ableiten, die ohne weiteres auf eine ganze Berufsgruppe übertragbar wären. Ziel der Umfrage war es lediglich, erste Hinweise für eine Einschätzung möglicher positiver und negativer Auswirkungen von digitalen und KI-Technologien zu geben, die dann ggfs. in weiteren Untersuchungen fokussiert werden sollten.

Insgesamt deuten die Ergebnisse der Umfrage überwiegend positive Effekte von Digitalisierung und KI-Technologien auf wissenschaftliche Arbeitstätigkeiten an. Obgleich digitale Technologien - wie in vielen anderen Berufsfeldern auch - die Work-Life-Balance ins Schwanken bringen, scheint dies nur von wenigen Forschenden als problematisch bewertet zu werden. Digitale Technologien werden von Forschenden mehrheitlich als Hilfsmittel angesehen, die das Potenzial besitzen, sowohl die Produktivität als auch die Qualität der wissenschaftlichen Arbeit zu steigern.

Wie in vielen Forschungsarbeiten zu künstlicher Intelligenz zeigt auch diese Umfrage unter Forschenden, dass der Begriff „KI“ für verschiedene Personen unterschiedliche Bedeutungen hat. Technologien, die gemäß der in der Umfrage verwendeten Arbeitsdefinition als KI-Technologie bezeichnet werden könnten, kommen zwar bislang nur wenig (zumindest bewusst) zum Einsatz, jedoch werden auch diese eher selten negativ bewertet. Wie so häufig in Bezug auf Automationstechnologien beobachtet, wird auch beim Einsatz von KI-Technologien eine mangelnde 
Transparenz und Nachvollziehbarkeit von Arbeitsprozessen und -ergebnissen befürchtet. Einschränkungen der wissenschaftlichen Selbstbestimmtheit oder des Forschungsalltags werden jedoch nur von wenigen befürchtet.

Es zeichnet sich ferner die Tendenz ab, dass Personen, die KI-Technologien häufiger einsetzen, den Technologien eher positive Auswirkungen zuschreiben. Dies könnte auf den Aspekt der freiwilligen Nutzung zurückzuführen sein: wer der Auffassung ist, dass KI-Technologien die Arbeit unterstützen, wird diese auch mit höherer Wahrscheinlichkeit bei sich einsetzen. Bei vielen digitalen Technologien ist diese Freiwilligkeit jedoch heutzutage nicht mehr gegeben: Forschende, die z. B. keine E-Mail nutzen oder nicht online publizieren, können ihre Aufgaben nicht im gleichen Maße erfüllen, wie Forschende, die diese Technologien nutzen. Sollte der Einsatz von KI-Technologien zukünftig für die wissenschaftliche Arbeit (und/ oder Akzeptanz in der Community) zwingend notwendig werden, könnte dies auch eine negativere Bewertung solcher Technologien bewirken.

\subsection{Fazit}

Bislang existieren kaum arbeitswissenschaftliche Untersuchungen $\mathrm{zu}$ den möglichen Auswirkungen von Digitalisierungsmaßnahmen und dem Einsatz von KIbasierten Technologien auf die wissenschaftliche Arbeit und die Arbeitsbedingungen von Wissenschaftlerinnen und Wissenschaftlern; entsprechend unsicherheitsbehaftet sind deren Prognosen. Lassen sich jedoch arbeitswissenschaftliche Erkenntnisse zur Wissensarbeit übertragen, kann davon ausgegangen werden, dass wissenschaftlich Arbeitende von dem aktuellen Entwicklungstrend in vielerlei Hinsicht profitieren. Digitale Technologien und KI fördern sowohl die Arbeitsproduktivität als auch -qualität und schaffen mehr Freiräume für kreative Innovationsarbeit - insbesondere durch die Automatisierung von ungeliebten Routinetätigkeiten. Erfolgt diese Automatisierung jedoch ohne systematische Berücksichtigung menschlicher Fähigkeiten und Bedürfnisse, werden wissenschaftliche Erkenntnisse selbst für die Forschenden zunehmend schwieriger nachzuvollziehen. Mögliche Folgen: Ergebnisse können nicht mehr repliziert oder kritisch hinterfragt werden und Forschende verlieren nicht nur die Möglichkeit, an Forschungsprozessen zu lernen, sondern auch die Arbeitsmotivation aufgrund mangelnder Selbstwirksamkeit. Für den Erhalt der physischen und psychischen Gesundheit von Forschenden ist es ferner unabdingbar, auf menschenverträgliche Arbeitsbedingungen zu achten. Ergonomisch gestaltete Arbeitsplätze (auch im Homeoffice!) und regelmäßige Arbeitspausen von ausreichender Dauer sind eine wichtige Voraussetzung hierfür.

Aus gesamtwirtschaftlicher Perspektive kann festgehalten werden, dass die häufig in den Medien geäußerten Befürchtungen einer Massenarbeitslosigkeit durch 
die Anwendung von KI-Systemen übertrieben erscheinen. Zwar werden KIAnwendungen in Zukunft in immer mehr Berufen die Menschen unterstützen. Das gilt auch für den Beruf der Wissenschaftler/Wissenschaftlerinnen. Allerdings wird es nur in wenigen Berufsfeldern zu einer Substitution kommen. Zudem ist bei der Analyse der Nettoeffekte auf dem Arbeitsmarkt auch zu berücksichtigen, dass neue Jobs entstehen werden. Gerade im Wissenschaftsbereich brauchen sich Forscherinnen und Forscher also keine Sorgen zu machen, dass KI-Anwendungen zukünftig ihre Arbeiten übernehmen werden. Insbesondere kreatives Denken oder die Entwicklung von Innovationen werden auf absehbare Zeit weiterhin Domäne der Menschen bleiben. KI-Anwendungen können aber Wissenschaftlerinnen und Wissenschaftler bei ihrer Arbeit unterstützen. Damit ist die Hoffnung verbunden, dass dies zu einer Steigerung der Effizienz und Effektivität ihrer Forschungstätigkeiten führen kann. Jedoch sind auch politische Maßnahmen gefragt, die nicht nur prekären Beschäftigungsverhältnissen, sondern auch einer KI-begünstigten Entwertung wissenschaftlicher Arbeit und der damit verbundenen Verlagerung von Arbeitsplätzen in Niedriglohnländer entgegenwirken.

Die vorliegenden Ergebnisse zeigen weiterhin einen großen Forschungsbedarf für die Arbeitswissenschaft in den kommenden Jahren auf. So existieren bislang zum Beispiel kaum Kenntnisse zu den Effekten von KI auf innovative Gruppenarbeit, eine Arbeitsform, die insbesondere in einigen Wissenschaftsdisziplinen weit verbreitet ist. Unklar ist ebenso, welche Merkmale Mensch-Maschine Schnittstellen aufweisen sollten, um sowohl die Handlungs- als auch Entscheidungskompetenz von Beschäftigten im Umgang mit KI-Technologien zu fördern und zu präservieren. Weiterhin besteht noch Unklarheit über neue Arbeitsaufgaben und Arbeitsplätze, die durch KI-Technologien geschaffen werden. Dies hat u. a. zur Folge, dass weder Bildungseinrichtungen noch Unternehmen einschätzen können, welche Kompetenzen Beschäftigte in den kommenden Jahren benötigen, um auch den zukünftigen Arbeitsaufgaben in einer digitalisierten Arbeitswelt gerecht zu werden.

\section{Literatur}

Adadi A, Berrada M (2018) Peeking inside the black-box: a survey on explainable artificial intelligence (XAI). IEEE Access 6:52138-52160. https://doi.org/10.1109/ACCESS.2018.2870052

Arlinghaus A (2017) Wissensarbeit: Aktuelle arbeitswissenschaftliche Erkenntnisse. Mitbestimmungsreport 35. Düsseldorf. http://hdl.handle.net/10419/175264. Zugegriffen am 13.10.2020

Arntz M, Gregory T, Zierahn U (2019) Digitization and the future of work: macroeconomic consequences. In: Zimmermann KF (Hrsg) Handbook of labor, human resources and population economics. Springer International Publishing, Cham, S 1-29

Autor DH, Price B (2013) The changing task composition of the US Labor Market: an update of Autor, Levy, and Murnane (2003). MIT, Arbeitspapier

Buxmann P (2020) Künstliche Intelligenz - Wirtschaft und Innovation. Studie im Auftrag des Ministeriums für Digitales für das Land Hessen. Wiesbaden. (im Erscheinen) 
Buxmann P, Schmidt H (Hrsg) (2020) Künstliche Intelligenz. Mit Algorithmen zum wirtschaftlichen Erfolg, 2. Aufl. Springer Gabler, Berlin

Drucker PF (1991) Management. An abridged and revised version of management: tasks, responsibilities, practices. Butterworth-Heinemann, Oxford

Eichhorst W (2015) Der Wandel der Erwerbsformen in Deutschland. IZA Standpunkte 78. Forschungsinstitut zur Zukunft der Arbeit (IZA), Bonn

Frey CB, Osborne MA (2013) The future of employment: how susceptible are jobs to computerisation? Oxford Martin 114. https://doi.org/10.1016/j.techfore.2016.08.019.

Frey CB, Osborne MA (2017) The future of employment: how susceptible are jobs to computerisation? Technol Forecast Soc Chang 114:254-280

Hacker W (2018) Menschengerechtes Arbeiten in der digitalisierten Welt. Eine Wissenschaftliche Handreichung, 1. Aufl. Mensch - Technik - Organisation, Bd 49. vdf Hochschulverlag, Zürich

Hirsch-Kreinsen H, Karačić A (2019) Autonome Systeme und Arbeit: Perspektiven, Herausforderungen und Grenzen der Künstlichen Intelligenz in der Arbeitswelt. transcript, Bielefeld

Hoppe A (2010) Komplexe Technik-Hilfe oder Risiko? Darstellung ausgewählter Ergebnisse einer Grundlagenuntersuchung zu Technikstress! In: Brandt C (Hrsg) Mobile Arbeit - Gute Arbeit? Arbeitsqualität und Gestaltungsansätze bei mobiler Arbeit. Gewerkschaft ver.di, Berlin, S 53-64

Köchling A (1985) Bildschirmarbeit. Gesundheitsregeln u. Gesundheitsschutz. Wissenschaft im Arbeitnehmerinteresse, Bd 3. Bund, Köln

May TY, Korczynski M, Frenkel SJ (2002) Organizational and occupational commitment: knowledge workers in large corporations. J Manag Stud 39(6):775-801

Moser K, Preising K, Göritz AS, Paul K (2002) Steigende Informationsflut am Arbeitsplatz: belastungsgünstiger Umgang mit elektronischen Medien (E-Mail, Internet), 1. Aufl. Bremerhaven: Wirtschaftsverlag NW Verlag für neue Wissenschaft GmbH, S 228. (Schriftenreihe der Bundesanstalt für Arbeitsschutz und Arbeitsmedizin: Forschungsbericht, Fb 967) ISBN: 3-89701-900-0, Projektnummer: F1814

Müller JC (2015) Arbeit - Macht - Sinn: Zur Entgrenzung von Arbeit im Wissenschaftsbetrieb. In: Krentel F, Barthel K, Brand S, Friedrich A, Hoffmann AR, Meneghello L, Müller JC, Wilke C (Hrsg) Library Life: Werkstätten kulturwissenschaftlichen Forschens. meson press, Lüneburg, S 37-75

Mütze-Niewöhner S, Nitsch V (2020) Arbeitswelt 4.0. In: Frenz W (Hrsg) Handbuch Industrie 4.0: Recht, Technik, Gesellschaft. Springer, Berlin/Heidelberg, S 1187-1217

Nerdinger FW, Blickle G, Schaper N (2014) Arbeits- und Organisationspsychologie, 3. Aufl. Springer-Lehrbuch/Springer, Berlin/Heidelberg

OECD (2018) Putting faces to the jobs at risk of automation. Policy brief on the future of work. OECD Publishing, Paris. http://www.oecd.org/els/emp/future-of-work/Automation-policybrief-2018.pdf. Zugegriffen am 13.10.2020

Rick V, Distelrath J, Nitsch V (2021) Digitalisierung und KI in der Wissenschaft: Auswertung der Begleitstudie zum Projekt „Digitale Arbeitswelten in Forschung und Entwicklung“. DOI 10.18154/RWTH-2021-01212 . https://publications.rwth-aachen.de/record/811477. Zugegriffen am 07.02.2021

SAE International (2016) Taxonomy and definitions for terms related to driving automation systems for on-road motor vehicles. https://doi.org/10.4271/J3016_201609

Schmidt H (2018) Wie Maschinen die Arbeit übernehmen. Netzökonom. https://www.netzoekonom.de/2018/10/13/wie-maschinen-die-arbeit-uebernehmen/. Zugegriffen am 13.10.2020

Simpson CW, Prusak L (1995) Troubles with information overload - moving from quantity to quality in information provision. Int J Inf Manage 15(6):413-425

Webb M (2019) The impact of artificial intelligence on the labor market. https://doi.org/10.2139/ ssrn.3482150. Zugegriffen am 13.10.2020

Wilkesmann U (2005) Die Organisation von Wissensarbeit. Berl J Soziol 15(1):55-72

Willke H (1998) Organisierte Wissensarbeit. Z Soziol 27(3):161-177 
World Economic Forum (2018) The future of jobs report 2018. Insight report. World Economic Forum, Cologny/Geneva

Zika G, Helmrich R, Maier T, Weber E, Wolter MI (2018) Arbeitsmarkteffekte der Digitalisierung bis 2035: Regionale Branchenstruktur spielt eine wichtige Rolle. IAB-Kurzbericht 9/2018. Nürnberg. http://hdl.handle.net/10419/185844. Zugegriffen am 13.10.2020

Open Access Dieses Kapitel wird unter der Creative Commons Namensnennung 4.0 International Lizenz (http://creativecommons.org/licenses/by/4.0/deed.de) veröffentlicht, welche die Nutzung, Vervielfältigung, Bearbeitung, Verbreitung und Wiedergabe in jeglichem Medium und Format erlaubt, sofern Sie den/die ursprünglichen Autor(en) und die Quelle ordnungsgemäß nennen, einen Link zur Creative Commons Lizenz beifügen und angeben, ob Änderungen vorgenommen wurden.

Die in diesem Kapitel enthaltenen Bilder und sonstiges Drittmaterial unterliegen ebenfalls der genannten Creative Commons Lizenz, sofern sich aus der Abbildungslegende nichts anderes ergibt. Sofern das betreffende Material nicht unter der genannten Creative Commons Lizenz steht und die betreffende Handlung nicht nach gesetzlichen Vorschriften erlaubt ist, ist für die oben aufgeführten Weiterverwendungen des Materials die Einwilligung des jeweiligen Rechteinhabers einzuholen. 